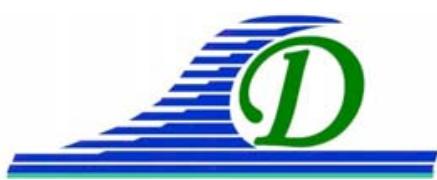

XIII ${ }^{\grave{m} e s}$ Journées Nationales Génie Côtier - Génie Civil

Dunkerque, 2-4 juillet 2014

DOI:10.5150/jngcgc.2014.066 (C) Editions Paralia CFL

disponible en ligne - http://www.paralia.fr - available online

\title{
Détection d'indicateurs géomorphologiques littoraux par télédétection à très haute résolution spatiale
}

\section{Virginie LAFON ${ }^{1}$, Aurélie DEHOUCK ${ }^{1}$, Arthur ROBINET ${ }^{1}$, Vincent MARIEU ${ }^{2}$, Cécile CURTI $^{1}$, Nicolas DUROU ${ }^{1}$}

\section{GEO-Transfert, UMR 5805 EPOC, al. Geoffroy St Hilaire, CS 50023, 33615 Pessac,}

France.

v.lafon@epoc.u-bordeaux1.fr ; a.dehouck@epoc.u-bordeaux1.fr

2. CNRS ; UMR 5805 EPOC, université de Bordeaux, allée Geoffroy St Hilaire, CS 50023, 33615 Pessac cedex, France.

v.marieu@epoc.u-bordeaux1.fr

\section{Résumé :}

Cette contribution dresse un bilan des développements méthodologiques évalués sur des données très haute résolution spatiale Pléiades et TerraSAR-X concernant la détection d'indicateurs géomorphologiques du trait de côte ; en particulier la morphologie sousmarine, le pied de dune et les faciès dunaires des littoraux sableux battus. Nous présentons ici des exemples d'applications sur les façades atlantiques et méditerranéennes où des observations de terrain indispensables à la validation des cartes produites ont pu être recueillies. Pour restituer la bathymétrie, nous couplons une approche dérivée de l'optique marine applicable aux eaux claires (pénétration de la lumière supérieure à $5 \mathrm{~m}$ ) à une méthode utilisant l'inversion des crêtes de houle afin d'aborder également des environnements plus turbides comme les embouchures de fleuves et les littoraux agités. Le pied de dune est détecté par analyse de texture (indices d'Haralick du premier ordre) avec une grande précision spatiale ( $<5 \mathrm{~m})$. Enfin, pour la cartographie du couvert végétal du cordon dunaire, la méthode développée couple plusieurs descripteurs temporels et texturaux extraits de deux images (printemps/été). Introduits dans un algorithme de classification, ces descripteurs permettent de distinguer la dune grise de la dune blanche et de classer correctement $92 \%$ des pixels.

Mots-clés : Télédétection, Pléiades, SAR, Bathymétrie, Trait de côte, Cartographie, Optique marine, Texture, Crêtes de houle.

\section{Introduction}

Dans le contexte du changement global et dans la perspective du développement durable des littoraux, un besoin croissant de cartographies est exprimé en appui aux gestionnaires et aux politiques publiques. Or, la télédétection spatiale montre toutes les qualités pour établir la cartographie d'indicateurs géomorphologiques du littoral permettant d'évaluer sa position et son évolution dans le temps (LAFON et al., 2004 ; 2010 ; KUMAR et al., 2010 ; DEHOUCK et al., 2012b ; FROIDEFOND \& LAFON, 
2012 ; CAPO et al., 2013) et ainsi d'analyser son état de conservation et sa vulnérabilité face à l'érosion et à la submersion. La multiplication de capteurs satellitaires à très haute résolution spatiale (THR), actifs ou passifs et dans les domaines spectraux optiques et radar, offre de nouvelles possibilités. La très faible taille des pixels permet notamment de discriminer des objets distants de moins d'un mètre et de fiabiliser les méthodes de détection, les pixels intégrant une variabilité radiométrique moindre.

Dans le cadre du projet APSAT, différentes approches de cartographie des littoraux sableux battus ont été inventoriées, améliorées, adaptées aux données THR, optimisées pour en faire une exploitation multi-sites et enfin validées. Le manuscrit sera organisé autour de quatre thématiques: la topographie sous-marine par inversion optique et inversion des crêtes de houle, le trait de côte et les faciès dunaires. Pour chacune d'elle, les méthodes, données et résultats seront détaillés. Ces parties centrales seront précédées par la présentation des sites d’études.

\section{Localisation des sites atelier}

Les différents sites atelier (Atlantique, Méditerranée) sont présentés sur la figure 1.

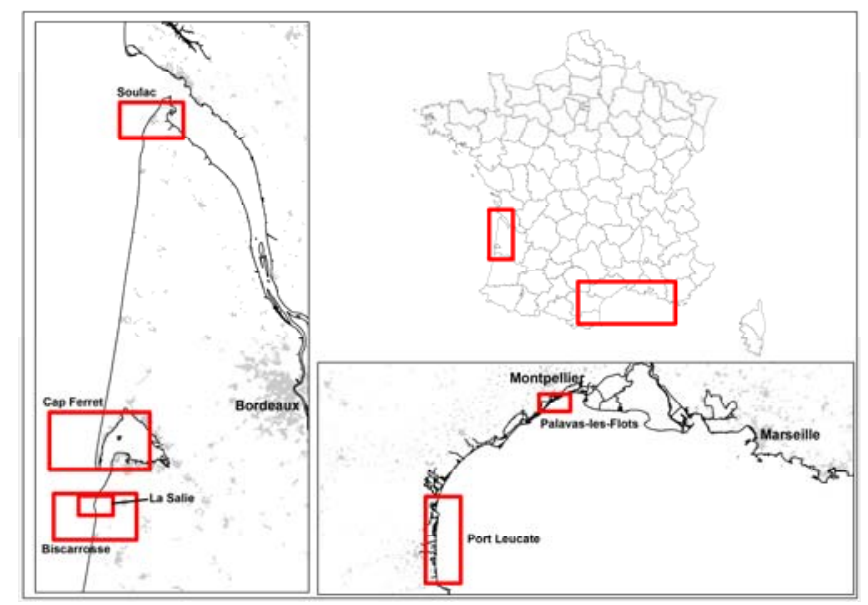

Figure 1. Localisation des sites atelier.

\section{Bathymétrie par inversion d'un code de transfert radiatif}

\subsection{Méthode}

Lorsque le fond est visible, l'élaboration de cartes bathymétriques par télédétection s'appuie sur les propriétés optiques des eaux littorales où la réflectance de surface s'exprime comme la somme des contributions du fond (albedo) et de la colonne d'eau (LYZENGA, 1978). L'utilisation de plusieurs bandes spectrales dans les modèles d'inversion est nécessaire pour retrouver la profondeur à moins de contrôler les variations de la turbidité et de l'albedo du fond, sur le terrain par exemple. Réservée aux sites accessibles, cette approche donne des résultats deux fois plus précis que ceux qui 


\section{XIII ${ }^{\text {èmes }}$ Journées Nationales Génie Côtier - Génie Civil \\ Dunkerque, 2-4 juillet 2014}

peuvent être obtenus sans paramétrisation comme cela a été démontré dans l'embouchure d'Arcachon (LAFON et al. 2002 ; CAPO et al., 2013). Afin de préserver la qualité des produits cartographiques, une méthode dérivée des travaux de LAFON et al. (2002) et DEHOUCK et al. (2009) est développée ici. L'information bathymétrique est retrouvée à l'issue des étapes suivantes: (i) corrections atmosphériques (code 6S, VERMOTE et al., 1997), (ii) conversion en réflectance de sub-surface, (iii) transformation en hauteur d'eau par l'application de l'algorithme semi-analytique de LEE et al. (1998) inversé suivant la formulation de BIERWIRTH et al. (1993). La paramétrisation de la relation d'inversion bathymétrique s'appuie sur des données propres à l'image (réflectance du fond et des eaux du large) et sur la détermination du coefficient d'atténuation de la lumière dans l'eau $(K)$, variable dans l'espace et dans le temps. Ce dernier est obtenu par ajustement empirique sur une imagette d'entrainement $\left(2,25 \mathrm{~km}^{2}\right)$ associée à quelques profils bathymétriques. La précision des résultats est évaluée à partir de paramètres statistiques suivants : erreur absolue moyenne (MAE), erreur relative moyenne (MRE) et erreur quadratique moyenne (RMSE).

\subsection{Données}

Une scène Pléiades du 22/07/2013 (Palavas-les-Flots), calée à partir de l'ortho-littorale (Lambert 93) a été traitée puis comparée à des sondes hydrographiques. Les mesures de la station AERONET de Toulon (épaisseur optique atmosphérique, nombre d'Angström, fraction d'aérosols fins, distribution en taille des aérosols) ont été utilisées en paramètres d'entrée de $6 \mathrm{~S}$ pour restituer le réflectance de l'eau, ensuite inversée en hauteur d'eau.

\subsection{Résultats et discussion}

La qualité de l'inversion des données optiques (tableau 1) diminue avec la profondeur : de $46 \mathrm{~cm}$ dans les 5 premiers mètres de la colonne d'eau, la MAE peut atteindre $94 \mathrm{~cm}$ entre 5 et $10 \mathrm{~m}$. Ces résultats sont un peu moins satisfaisants que ceux obtenus sur d'autres sites avec des données SPOT. En effet, à l'embouchure d'Arcachon, la MAE est de l'ordre de $30 \mathrm{~cm}$ dans les 6 premiers mètres de la colonne d'eau (LAFON et al., 2002). Un test réalisé sur le littoral du Roussillon (Port Leucate) dans le cadre du projet APSAT montre des résultats équivalents pour les 10 premiers mètres de la colonne d'eau (cf. tableau 1).

Les moins bons résultats sur l'image Pléiades peuvent être attribués à la réflexion spéculaire de la lumière par la surface de l'eau. Les données d'Arcachon et du Roussillon en sont dénuées. Des méthodes sont actuellement testées (KAY et al., 2009) afin d'atténuer ces effets et de réaliser une inversion en profondeur plus précise. 
Thème 3 - Instrumentation, mesures, imagerie et télédétection

Tableau 1. Validation des profondeurs inversées à partir des données Pléiades (Palavas-les-Flots) et SPOT (Port Leucate).

\begin{tabular}{lllllllll}
\hline \multirow{2}{*}{$\begin{array}{l}\text { Profondeur } \\
(\boldsymbol{m})\end{array}$} & $\boldsymbol{M A E}(\boldsymbol{m})$ & \multicolumn{3}{c}{$\boldsymbol{R M S E}(\boldsymbol{m})$} & \multicolumn{2}{c}{ MRE (\%) } & \multicolumn{2}{c}{ Pts validation } \\
\cline { 2 - 9 } & Pléiades & SPOT & Pléiades & SPOT & Pléiades & SPOT & Pléiades & SPOT \\
\hline $0-5$ & 0,46 & 0,27 & 0,65 & 0,34 & 15,7 & 12,2 & & \\
$5-10$ & 0,94 & 0,59 & 1,20 & 0,78 & 13,4 & 8,1 & & \\
$0-10$ & 0,70 & 0,45 & 0,96 & 0,63 & 14,5 & 9,8 & 23684 & 15536 \\
\hline
\end{tabular}

\section{Bathymétrie par inversion des crêtes de houle}

\subsection{Méthode}

Dans des zones plus turbides et agitées, l'inversion des crêtes de houle est une méthode qui apporte des résultats encourageants pour restituer la profondeur (LEU \& CHANG, 2005 ; PLESKASHEVSKY et al., 2011 ; RABAUTE et al., 2013). Dans ces différentes contributions, les longueurs d'onde des vagues sont obtenues par transformée de Fourrier bidimensionnelle (FFT 2D), à laquelle est substituée ici une approche par corrélation 2D. Celle-ci consiste à corréler une sous-image de référence avec des sousimages voisines dans la direction des vagues, cette dernière étant calculé par transformée de radon. Le maximum de corrélation correspond à une translation d'une longueur équivalente à une longueur d'onde des vagues. Cette approche présente l'avantage de pouvoir rejeter, au cours du calcul, les longueurs d'onde associées à un faible coefficient de corrélation. Puis, une technique itérative basée sur le suivi des lignes de vagues (PLESKASHEVSKY et al., 2011) permet l'application de cette méthode à l'ensemble du champ de houle présent sur les images. Enfin, la profondeur est calculée par inversion de l'équation de dispersion des vagues dans laquelle l'effet du courant est négligé (HOLLAND, 2001), qui lie la profondeur à la longueur d’onde des vagues (mesurée sur l'image) et à leur période (bouée, simulation). Ici, nous considérons les périodes et longueurs d’onde pic.

\subsection{Données}

Cette stratégie a été appliquée à une image TerraSAR-X du 21/06/2010, géoréférencée et projetée en Lambert 2 Etendu. Les sondages de référence (avril à juin 2010) sont calés par rapport au zéro hydrographique. La hauteur d'eau liée à la marée au moment de la prise de vue provient du modèle du SHOM (Biscarrosse plage). Enfin, la période pic des vagues au moment de la prise de vue est prédite par le modèle WaveWatch III.

\subsection{Résultats et discussion}

Les erreurs sont estimées pour des tranches de profondeur allant 0 à 35 m (tableau 2). L'erreur relative (MRE) est stable et représente correctement l'imprécision de cette approche bathymétrique, imprécision qui augmente avec la profondeur comme le 


\section{XIII ${ }^{\text {èmes }}$ Journées Nationales Génie Côtier - Génie Civil \\ Dunkerque, 2-4 juillet 2014}

montrent les valeurs de la MAE et RMSE calculées. Bien que les RMSE soient plus importantes que celles obtenues par inversion optique, l'inversion des crêtes de houle est une méthode complémentaire car elle fonctionne dans des eaux turbides, pour des gammes de profondeur plus importantes et sans donnée de calage. La plupart des erreurs mesurées sont vraisemblablement causées par une imprécision sur la valeur de période pic utilisée et par l'hétérogénéité du champ de houle.

Tableau 2. Validation des profondeurs mesurées par inversion des crêtes de houle à l'embouchure d'Arcachon.

\begin{tabular}{lllll}
\hline Profondeur $(\boldsymbol{m})$ & MAE $(\boldsymbol{m})$ & RMSE $(\boldsymbol{m})$ & MRE (\%) & Pts validation \\
\hline $0-5$ & 0,78 & 0,96 & 25,2 & 975 \\
$0-15$ & 1,42 & 1,93 & 24,4 & 2163 \\
$0-35$ & 2,45 & 3,70 & 25,5 & 2727 \\
\hline
\end{tabular}

L’imprécision de la méthode dérivée de la FFT 2D est plus importante que celle obtenue par calcul de corrélations (RABAUTE et al., 2013), ce qui démontre l'efficacité d'imposer un critère de qualité lors de la détermination des longueurs d'onde et d'éliminer les plus douteuses. Ainsi, seules les longueurs d'onde du champ de houle les plus fiables sont calculées. Toutefois, cette distribution discontinue des longueurs d'onde produit une bathymétrie qui doit être interpolée pour aboutir à un MNT complet. L'utilisation de plusieurs images satellites acquises à quelques jours d'intervalle permettrait de pallier les zones non détectées et pour les zones superposables de calculer les profondeurs avec une plus grande précision, par cumul de données.

\section{Détection de traits de côtes}

\subsection{Méthode}

Sur les images THR, le pied de dune est souvent bien marqué par des effets d’ombre. La méthode de détection développée repose sur une approche de classification très simplifiée qui exclut l'eau (seuil dans le proche infrarouge) et la forêt (seuillage de l'image de NDVI, ROUSE et al., 1973), couplée à un algorithme itératif qui repère successivement des discontinuités significatives dans les images de texture issues de la matrice de cooccurrence d'HARALICK et al., 1973 (indicateurs de premier ordre). Puis une fenêtre glissante ( 3 lignes $\times 6$ colonnes) repère successivement trois discontinuités dans les valeurs d'écart type et/ou de moyenne: (i) le premier pixel valide, (ii) le premier pixel de la plage, (iii) le premier pixel de sable sec, (iv) le pied de dune. Après chaque étape, des détections erronées (points ou couples de points isolés) sont recalées. Enfin, les points détectés sont reliés en une polyligne. Afin de valider la qualité du traitement automatique les polylignes brutes ont été superposées et comparées à des observations DGPS ou GPS in situ. Une ultime étape est proposée au traitement des 
images qui vise à vérifier le trait de côte par photo-interprétation par rapport à l'image panchromatique correspondante et repositionner les sommets de la polyligne spatialement décalés, du fait d’un défaut de détection de notre algorithme itératif. Une nouvelle validation est proposée pour cette polyligne recalée.

\subsection{Données}

Des images Pléiades (recalées et projetées en Lambert 93) du Cap Ferret (03/02/2012, 14/04/2013), Biscarrosse (26/06/2012), Soulac (06/11/2012) et La Salie (13/04/2013) (cf. figure 1) ont été traitées et comparées à des observations DGPS.

\subsection{Résultats}

Pour valider le trait de côte brut, 302 points de contrôle ont été utilisés. Nous constatons que $74 \%$ des points sont situés à moins de $10 \mathrm{~m}$ du levé de terrain, valeur qui correspond à l'erreur moyenne obtenue par traitement semi-automatique des données Formosat-2 sur le littoral aquitain (LAFON et al., 2010). L'erreur moyenne de la détection est de 14,3 m avec un biais de $3 \mathrm{~m}$ vers l'est (contre $6 \mathrm{~m}$ vers l'ouest pour Formosat-2). Dans le cas du trait de côte recalé, nous avons considéré 295 points de contrôle. L'exercice est concluant : $93 \%$ des points sont situés à moins de $10 \mathrm{~m}$ de la vérité terrain. La RMS étant de 4,5 m pour un biais absolu de 0,5 m vers l'est. L'effort de recalage a été chiffré. Sur les 6049 points générés par le traitement automatique, près de 60 \% d'entre eux ne nécessitent aucune correction. Les 40\% restant sont recalés sous ArcGIS afin de produire une polyligne dont la précision est garantie à $5 \mathrm{~m}$.

\section{Détection des faciès dunaires}

\subsection{Méthode}

Parmi les indicateurs géomorphologiques les plus pertinents en zone dunaire, la dune blanche et la dune grise sont difficiles à distinguer par classification d'une scène unique (LAFON et al., 2010). Pour pallier cette difficulté, une approche multi-temporelle simple (DEHOUCK et al., 2012a) a été adaptée pour augmenter le nombre de classes et de faciès dérivés des données spatiales. Différentes combinaisons d'images ont été testées. Des descripteurs plus classiques ont également été pris en compte (indices de végétation, images issues de la matrice de cooccurrence). Les descripteurs les plus utiles ont été rassemblés dans un arbre de décision, et la carte produite validée à l'aide de données de terrain et/ou de données photo-interprétées.

\subsection{Données}

La méthode de cartographie des faciès a été testée sur le site de La Salie au sud de la Gironde et sur des images acquises en juin, août, novembre 2012 et avril 2013. 


\section{XIII ${ }^{\text {èmes }}$ Journées Nationales Génie Côtier - Génie Civil \\ Dunkerque, 2-4 juillet 2014}

\subsection{Résultats}

L'indice NDVI permet de classer systématiquement l'océan (dans notre cas : eaux très peu turbide et littoral sableux battu : NDVI $<0$ ) et la forêt (NDVI $>0,3)$. Entre les deux apparaissent sans discrimination les autres faciès. Sur la plage, le sable humide adopte une signature spectrale typique, on le met en évidence par un seuil sur la bande rouge. La dune grise peut également être mise en évidence sur l'image d'avril 2013 à l'aide du NDVI, dont les valeurs sont comprises entre 0,11 et 0,3. Des tests menés dans la base de données Pléiades, afin de retrouver cette caractéristique à d'autres dates, montrent que ce descripteur fonctionne également bien sur les images du 03/02/2012 et du 14/04/2013 au Cap Ferret. Plus généralement dans la base de données Kalideos, les images acquises en mai 2010 et mars 2011 montrent un renforcement du NVDI sur la dune grise, contrairement à la scène de mars 2009 où les NDVI sont très faibles sur l'ensemble du secteur dunaire. Entre juin et novembre, le NDVI de la dune blanche augmente et est apparemment partiellement confondu avec celui de la dune grise.

Pour différencier la plage et la dune blanche (mosaïque de pixels de sable nu et plus ou moins couvert de végétation) on utilise un descripteur lié à la texture. En effet, dans la matrice de cooccurrence la variance radiométrique de la plage est beaucoup plus faible que celle de la dune blanche. C’est cette propriété qui est exploitée pour détecter le pied de dune. Mais on utilise cette fois le descripteur dans les deux dimensions de l'espace pour différencier la plage de la dune. Le descripteur sélectionné est l'image de variance de la bande verte de la matrice de cooccurrence calculée sur des fenêtres de 9x9 pixels. La combinaison de deux scènes (août ou novembre 2012 et avril 2013) donne des résultats de classification bien meilleurs que ceux obtenus à partir des seules données Formosat-2 (LAFON et al., 2010). La précision globale de la carte passe en effet de $73 \%$ à près de $92 \%$. En outre, l'objectif de discrimination entre dune blanche et grise est atteint. En effet, près de 70 et $80 \%$ dans pixels sont correctement classés dans ces deux cas, respectivement.

\section{Synthèse et conclusions}

Les images Pléiades et TerraSAR-X acquises sur différents littoraux métropolitains ont permis de réaliser d'importants progrès méthodologiques concrétisés par des résultats très encourageants dans trois domaines : la bathymétrie des petits fonds sableux, la détection du trait de côte (pied de dune) et la cartographie du couvert végétal des cordons dunaires.

La bathymétrie est alternativement obtenue par inversion d'un modèle de transfert radiatif ou par inversion des crêtes de houle. L'extraction du trait de côte à partir des données Pléiades est un protocole comprenant: une analyse automatique basée sur l'analyse de la texture, une vérification à dire d'expert des points extraits du traitement, la constitution d'une polyligne basée sur les points validés à dire d'expert dont la précision est garantie à $5 \mathrm{~m}$. En outre l'algorithme que nous avons développé est 
exploitable pour extraire la position d'un autre indicateur du trait de côte : la limite du swash (limite sable sec / sable humide) utilisé par exemple sur le littoral méditerranéen Pour la cartographie des faciès en domaine dunaire, en combinant dans un arbre de décision associant trois descripteurs et deux scènes (printemps + été ou automne), près de $92 \%$ des pixels sont correctement classés et permettent de discriminer la plage, la dune blanche, la dune grise et la forêt, indicateurs géomorphologiques complémentaires au pied de dune.

Ces très bons résultats montrent que les données satellites à très haute résolution spatiale sont adaptées à la surveillance du littoral et, dans certains cas, permettent même une caractérisation géophysique de celui-ci bien meilleure qu'avec les précédents capteurs spatiaux. Ces résultats sont d'autant plus pertinents pour l'aide à la décision que les méthodes développées sont reproductibles. Ainsi elles peuvent participer à l'élaboration de stratégies de gestion du trait de côte de l'échelle nationale aux échelles territoriales.

\section{Remerciements}

Nous remercions le programme INTERREG SUDOE IVB qui a financé le projet APSAT et le CNES (programmes RTU, ISIS et Kalideos). Les données de validation proviennent de la DREAL Languedoc Roussillon, de la DDTM 33/SIBA, du laboratoire EPOC et de l'Observatoire de la Côte Aquitaine.

\section{Références bibliographiques}

BIERWIRTH P. N., LEE T. J., BURNE R. V. (1993). Shallow Sea-Floor Reflectance and Water Depth Derived by Unmixing Multispectral Imagery, Photogrammetic Engineering \& Remote Sensing. Vol. 59(3), pp 331-338.

CAPO S., MARIEU V., BRU D., LUBAC B., BONNETON P. (2013). Decadal morphodynamics evolution of a mixed-energy inlet using multispectral spot imagery. Coastal Dynamics 2013, pp 283-294.

DEHOUCK A., MARTINY N., FROIDEFOND J.-M., SENECHAL N., BUJAN S. (2009). New outcomes from spatial remote sensing during the ECORS experiment: towards validation of ocean color products and large-scale bathymetry mapping in a coastal zone. Journal of Coastal Research, Vol. SI 56 (T. 2), pp 1756-1760.

DEHOUCK A., LAFON V., BAGHDADI N., MARIEU V. (2012). Use of optical and radar data in synergy for mapping intertidal flats and coastal salt-marshes (Arcachon lagoon, France). IGARSS 2012, München, Germany, pp 2853-2856.

DEHOUCK A., SÉNÉCHAL N., LAFON V., FROIDEFOND J.-M., ALMAR R., CASTELLE B., MARTINY N. (2012B). Evolution morphodynamique interannuelle a decennale du littoral sud de la Gironde. RFPT, n 197, pp 31-42. 


\section{XIII ${ }^{\text {èmes }}$ Journées Nationales Génie Côtier - Génie Civil \\ Dunkerque, 2-4 juillet 2014}

FROIDEFOND J.-M., LAFON V. (2012). Surveillance de la morphologie sous-marine par télédétection spatiale: application aux passes d'entrée du bassin d'Arcachon. Revue Française de Photogrammétrie et Télédétection, n 197, pp 43-51.

HARALICK R.M., SHANMUGAN K., DINSTEIN I. (1973). Texture features for image classification. IEEE Trans. Syst., Man and Cyb., Vol. SMC-3(6), pp 610-621. http://dx.doi.org/10.1109/TSMC.1973.4309314

HOLLAND T. K. (2001). Application of the Linear Dispersion Relation with Respect to Depth Inversion and Remotely Sensed Imagery. IEEE Transactions on Geoscience and remote sensing, Vol. 39(9), September 2001, pp 2060-2072. http://dx.doi.org/10.1109/36.951097 KAY S., HEDLEY J.D., LAVENDER S. (2009). Sun Glint Correction of High and Low Spatial Resolution Images of Aquatic Scenes: a Review of Methods for Visible and Near-Infrared Wavelengths. Remote Sensing, Vol. 1, pp 697-730. http://dx.doi.org/10.3390/rs1040697

KUMAR A., NARAYANA A.C., JAYAPPA K.S. (2010). Shoreline changes and morphology of spits along southern Karnataka, west coast of India: a remote sensing and statistics-based approach. Geomorphology, Vol. 120(3-4), pp 133-152. http://dx.doi.org/10.1016/j.geomorph.2010.02.023

LAFON V., FROIDEFOND J.-M., LAHET F., CASTAING P. (2002). SPOT shallow water bathymetry of a moderately turbid tidal inlet based on field measurements, Remote Sensing of Environment, Vol. 81, pp 136-148. http://dx.doi.org/10.1016/S00344257(01)00340-6

LAFON V., DE MELO APOLUCENO D., DUPUIS H., MICHEL D., HOWA H. (2004). Morphodynamics of nearshore rhythmic sandbars in a mixed-energy environment (SW France): 1. Mapping beach changes using visible satellite imagery. Estuarine, Coastal and Shelf Science, Vol. 61, pp 289-299. http://dx.doi.org/10.1016/j.ecss.2004.05.006

LAFON V., HOAREAU A., MALLET C., DESPRATS J.-F. (2010). Suivi du trait de côte en Aquitaine par imagerie Formosat-2. $\mathrm{X}^{\text {èmes }}$ Journées Nationales Génie Côtier Génie Civil, Sables d’Olonne, pp 497-504. http://dx.doi.org/10.5150/jngcgc.2010.058-L

LEU L. G., CHANG H. W. (2005). Remotely sensing in detecting the water depths and bed load of shallow waters and their changes. Ocean Eng., Vol. 32, pp 1174-1198. http://dx.doi.org/10.1016/j.oceaneng.2004.12.005

LYZENGA D.R. (1978). Passive remote sensing techniques for mapping water depth and bottom features. Applied Optics, Vol. 17, pp 379-383. http://dx.doi.org/10.1364/AO.17.000379

LEE Z., CARDER K. L., MOBLEY C. D., STEWARD R. G, PATCH J. R. (1998). Hyperspectral remote sensing for shallow waters: I. A semianalytical model. Applied Optics Vol. 37(27), pp 6329-6338. http://dx.doi.org/10.1364/AO.37.006329

PLESKACHEVSKY A., LEHNER S., HEEGE T., MOTT C. (2011). Synergy and fusion of optical and synthetic aperture radar satellite data for underwater topography 
Thème 3 - Instrumentation, mesures, imagerie et télédétection

estimation in coastal areas. Ocean Dynamics, Vol. 61(12), pp 2099-2120. http://dx.doi.org/10.1007/s10236-011-0460-1

RABAUTE T., LAFON V. DEHOUCK A., ROBINET A., MARIEU V. (2013). Exploiting TerraSAR- $x$ data in the frame of the kalideos programme: methodological approach and examples. TerraSAR-X Science meeting, Oberpfaffenhofen, Germany.

ROUSE J. W., HAAS R. H., SCHELL J. A., DEERING, D. W. (1973). Monitoring vegetation systems in the Great Plains with ERTS. In 3rd ERTS Symposium, NASA SP351 I, pp 309-317

VERMOTE E. F., TANRE D., DEUZE J. L., HERMAN M., MORCRETTE J. J. (1997). Second simulation of the satellite signal in the solar spectrum: An overview. IEEE Transaction in Geoscience and Remote Sensing, Vol. 35(3), pp 675-686. http://dx.doi.org/10.1109/36.581987 